\title{
The Five Different Faces of the Diatoms
}

\author{
Monika Bojko' ${ }^{1}$, Dariusz Latowski ${ }^{1}$ \\ ${ }^{1}$ Department of Plant Physiology and Biochemistry, Faculty of Biochemistry, Biophysics and Biotechnology Jagiellonian \\ University Gronostajowa 7, 30-387 Kraków, Poland \\ m.bojko@uj.edu.pl; dariusz.latowski@uj.edu.pl
}

\begin{abstract}
Diatoms are one of the most important groups of photosynthetic microorganisms ubiquitous in all aquatic ecosystems. These inconspicuous organisms have achieved spectacular ecological success and furthermore, are used as model organisms in scientific research in the field of biochemistry and genetics. Their ecological success is provided by their ability to tolerance and fast acclimation to rapid changing environmental conditions. Ecological significance and the ease of culture facilitate investigation and attempts of explanation the mechanisms of processes occurring in these organisms on the physiological, biochemical and genetic level. This review describes a number of our investigation strategies and techniques to understand the phenomenon of these organisms. We focused on five important aspects of diatoms biology, presented here as the five different faces of these algae. Acclimation mechanisms to environmental factors (temperature, light, heavy metals, age of the culture), on physiological and biochemical level at short and long time exposition of diatoms, are presented as the first diatoms face. The second face presents diatoms as organisms useful for phytoremediation. The results of the molecular analyses of the selected enzymes involved in photoprotective mechanisms of diatoms is the third face of these algae. The significance of diatoms as a treasury of bioactive compounds (xanthophylls and long chain polyunsaturated fatty acids) important in human diet, is displayed as the fourth face. Finally, as the only one which has not been investigated in our laboratory, we describe the fifth face of diatoms - the rich source of biofuels. In our research we used two model diatoms species: Phaeodactylum tricornutum and Thalassiosira pseudonana.
\end{abstract}

Keywords: Diatoms, Phaeodactylum tricornutum, Thalassiosira pseudonana, Acclimation, Photosynthesis, Xanthophylls, LC-PUFA, Heavy metals, Biofuels.

\section{Introduction}

Diatoms (Heterokontophyta, Bacillariophyceae) are the dominant group of microalgae in fresh or seawater ecosystems. This important group of photosynthetic organisms fix 20 billion tons of carbon per year, which corresponds to $20 \%$ of global and $40 \%$ of marine net primary production $[1,2]$. These inconspicuous organisms achieved ecological success by their ability to acclimation to stressful and rapid changes of environmental conditions. On the other hand, the ease of diatoms culture facilitate investigation and explanation the mechanisms of processes occurring in these organisms on the physiological, biochemical and genetic level. Eventually, the newly acquired knowledge enables the use of diatoms in other fields of science or apply in industrial production.

This review present a number of our investigation strategies and techniques to understand the phenomenon of this organisms. The experiments were performed using two model diatom species: Phaeodactylum tricornutum CCAP/1055/1 and UTEX 645 strain and Thalassiosira pseudonana with known genome sequence. We focused on five important aspects of diatoms biology, presented here as the five different faces of these algae.

\section{The first face - Acclimation mechanisms of diatoms to environmental factors}

There is only few data about the tolerance and adaptation mechanisms of diatoms to changing environmental conditions, despite their widespread occurrence in nature. The greenhouse effect and fast temperature changing of water, strongly influence on physiology, especially photosynthesis of phytoplankton which results in $\mathrm{CO}_{2}$ fixation and finally biomass production $[1,2]$. We analysed the short $(120 \mathrm{~min})$ and long-time $(6 \mathrm{month})$ effect of the increasing temperature and light intensity on physiology and biochemistry on P. tricornutum and T. pseudonana.

We found that the activity of photosynthetic membranes of tested diatoms depends on physical properties of the membrane. The structure and composition of diatoms chloroplasts are different from chloroplast of vascular plants. The diatom chloroplasts are surrounded by four membranes and certain regions of thylakoid membrane are arranged in group. 
Thylakoid membranes are characterized by different pigments, proteins and lipids composition. The light harvesting systems of diatoms bind high amount of fucoxanthin (Fx), diadinoxanthin (Ddx) and diatoxanthin (Dtx) (details about diadinoxanthin cycle see chapter 4). Moreover, their chloroplasts contain chlorophylls a (chl a) and c (chls c) [3]. Diatoms membranes are composed of the same lipid classes as vascular plants, but fatty acids profile of these lipids is also unique and contain long-chain polyunsaturated fatty acids (LC-PUFA)[4].

Photosynthesis has to be regulated by fast reaction mechanisms to adapt to numerous changes of environmental factors such as temperature and light fluctuation. The physical properties of the photosynthetic membrane depend of interaction of different membrane components, such as proteins, lipids and pigments. The carotenoids play one of the most important roles in changing physiological properties of the membranes [5]. Our experiments indicate that diadinoxanthin de-epoxidation play new, important role in the short-term stabilisation of diatom photosynthetic membranes exposed to increased temperature (up to $40^{\circ} \mathrm{C}$ ) [6]. The results show, that the changes of the membrane during the time of course of de-epoxidation, as well as rigidifying properties of Dtx (product of de-epoxidation) result in temporary stabilisation of fluidity (S parameter, EPR methods) of peripheral and internal part of the membrane and stable rigidification of hydrophobic core of the membrane (Fig. 1). These processes affect the thermal stabilisation of the thylakoid membrane.

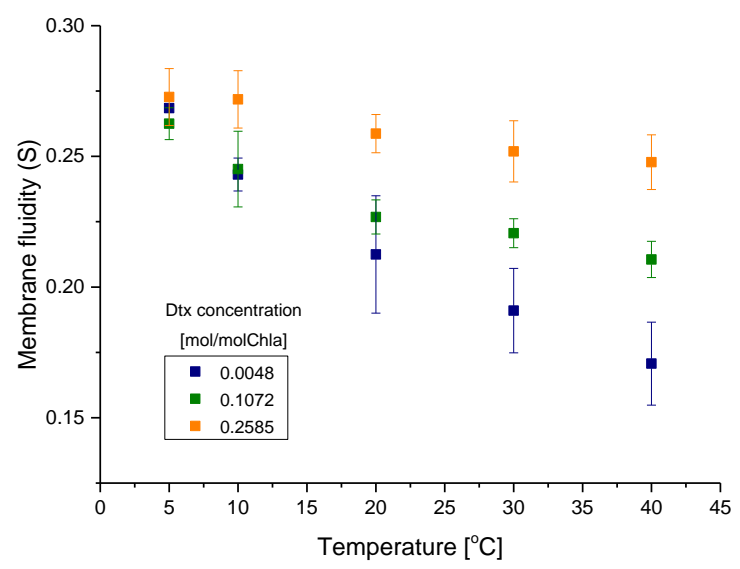

Fig. 1: Changes of interior thylakoid membranes of $P$. tricornutum fluidity (S, analysed for 16-SASL spin label by EPR methods) at different Dtx concentration (mol/mol Chla) against temperature (modified Bojko et al. 2019 [6]).

Long-time temperature effect on physiology and biochemistry of diatoms has been analysed for almost 6 months. During the first two weeks of the diatom cultures growth at low and moderate temperatures $\left(12\right.$ and $\left.20^{\circ} \mathrm{C}\right)$, the decrease of the cell proliferation (measured as optical density, $\mathrm{OD}_{600}$ ), as well as the chlorophylls content with increasing of the protein concentration, were observed. We also observed the increase of polyunsaturated and decrease of saturated fatty acids content (analysed by gas chromatography, GC), which resulted in stabilisation of the thylakoid membrane fluidity (S parameter, analysed by EPR method) and photosynthetic activity (Fv/Fm) [7, 8].

Table 1: Effect of temperature on physiological and biochemical parameters of T. pseudonana (modified Bojko et al. 2017 [8]).

\begin{tabular}{|c|c|c|c|c|c|c|c|c|}
\hline $\begin{array}{c}\text { Temperature } \\
{\left[{ }^{\circ} \mathbf{C}\right]}\end{array}$ & OD600 & $\begin{array}{l}\text { Protein } \\
{[\mu \mathrm{g} / \mathrm{ml}]}\end{array}$ & $\begin{array}{c}\text { Chlorophyll } \\
{[\mu \mathrm{g} / \mathrm{ml}]}\end{array}$ & Fv/Fm & $\begin{array}{c}\mathrm{S} \\
\text { at } 20^{\circ} \mathrm{C}\end{array}$ & PUFA & SFA & Time \\
\hline 12 & $\begin{array}{c}0.401 \\
( \pm 0.019)\end{array}$ & $\begin{array}{c}33.095 \\
( \pm 3.453)\end{array}$ & $\begin{array}{c}2.258 \\
( \pm 0.054)\end{array}$ & $\begin{array}{c}0.51 \\
( \pm 0.025)\end{array}$ & $\begin{array}{c}0.18393 \\
( \pm 0.0041)\end{array}$ & $\begin{array}{c}31.38 \\
( \pm 1.81)\end{array}$ & $\begin{array}{c}33,23 \\
( \pm 3.76)\end{array}$ & \multirow{2}{*}{$\begin{array}{l}12^{\text {th }} \\
\text { day }\end{array}$} \\
\hline 20 & $\begin{array}{c}0.48 \\
( \pm 0.026)\end{array}$ & $\begin{array}{l}27.443 \\
( \pm 3.95)\end{array}$ & $\begin{array}{c}2.755 \\
( \pm 0.409)\end{array}$ & $\begin{array}{c}0.48 \\
( \pm 0.034)\end{array}$ & $\begin{array}{c}0.18138 \\
( \pm 0.0065)\end{array}$ & $\begin{array}{c}25.35 \\
( \pm 2.23)\end{array}$ & $\begin{array}{c}37.69 \\
( \pm 3.92)\end{array}$ & \\
\hline
\end{tabular}

The results show that regulation of membrane fluidity by interaction of fatty acids, proteins and pigments is the most important factor in adaptation strategies of diatoms to environmental temperature changes (Tab.1). However, the results obtained during long time temperature changes (6 months) indicate that adaptation strategies to long periods of the 
moderate temperature is manifested only by cells proliferation slow down, whereas photosynthetic activity and photosynthetic pigments concentration (chlorophylls and diadinoxanthin cycle xanthophylls) are more sensitive to light conditions, than temperature increase [9].

\section{The second face - Diatoms in phytoremediation}

Heavy metals pollution of both soil and water are the rising environmental problem. The contaminants may be introduced to environment from industrial sewage, mining or using phosphate fertilizers. Heavy metals may be dangerous for many organisms including humans, but also for plants and algae [10,11,12,13]. Algae are very promising organisms which can be used for remediation of water contaminated by heavy metals. We investigated the resistance and ability to remove of selected heavy metals from environment by marine diatom $P$. tricornutum. The effect of cadmium(II), chromium(IV), mercury(II), thallium(I) and arsenic(V) were analysed spectrophotometrically as a change in optical density of culture at wavelength of $600 \mathrm{~nm}\left(\mathrm{OD}_{600}\right)$. We found that $P$. tricornutum can grow in culture media containing tested heavy metals and that efficiency of cell proliferation and resistance depend on the metal and its concentration (Fig.2).

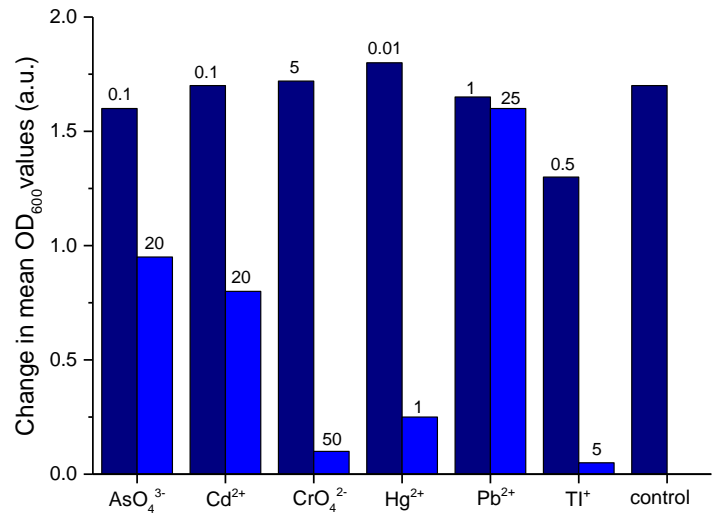

Fig. 2: The absolute change in mean $\mathrm{OD}_{600}$ (between the initial and the end phase of experiment) for $P$. tricornutum growth in control and in supplemented by different concentration of toxic compounds (heavy metal) (modified Tokarek 2016 [14], Listwan 2018 [15]). The concentrations of toxic compounds are given in $\mathrm{mg} / \mathrm{L}$ above bars.

Table 2: Comparison of concentration of toxic elements in the medium at the beginning and at the end of $P$. tricornutum culture (modified Listwan et al. 2018 [15]).

\begin{tabular}{|ll|c|c|}
\hline \multicolumn{2}{|c|}{$\begin{array}{c}\text { theoretical initial } \\
\text { concentration }[\mathbf{m g} / \mathbf{L}]\end{array}$} & $\begin{array}{c}\text { detected concentration at } \\
\text { the beginning of culture }\end{array}$ & $\begin{array}{c}\text { detected concentration at } \\
\text { the end of the culture }\end{array}$ \\
\hline arsenic & 0.4 & $0.329( \pm 0.0106)$ & $0.1361( \pm 0.0211)$ \\
\hline cadmium & 0.1 & $0.1209( \pm 00.0019)$ & $0.0549( \pm 0.0037)$ \\
\hline mercury & 0.01 & $0.01099( \pm 0.00007)$ & $0.00769( \pm 0.00002)$ \\
\hline
\end{tabular}

In lower concentration of cadmium, mercury and arsenic $P$. tricornutum showed ability to reduce concentration of those elements in culture medium (Tab. 2). The mechanism reducing mercury concentration was described [16], however mechanism reducing arsenic and cadmium concentration require more studies.

Additionally, we tested $P$. tricornutum growth in freshwater from polluted rivers [15]. We observed the reduction of growth of diatoms in polluted water comparing to control medium. However, the results show that the tested diatom species can grow in fresh water polluted by heavy metals. Obtained results indicate potentially beneficial role of $P$. tricornutum in bioremediation of contaminated water in future. 


\section{The third face - Molecular analyses of diatoms selected enzymes}

In spite of diatoms enormous ecological significance their success is not well understood. One of the most important processes is self-protection from photooxidative damage in fluctuating light condition in aquatic environment. The main photoprotective mechanism was controlled by the inter-conversion of epoxidised to de-epoxidised form of xanthophylls during process called xanthophyll cycle [17]. In diatoms, on molecular level, Ddx is de-epoxidised to Dtx by one step reaction catalysed by enzyme named diadinoxanthin deepoxidase. In the past in diatoms only one deepoxidase was known [18]. The significance of diatoms in marine ecosystem leads to sequencing of nuclear, plastid and mitochondrial genomes of centric diatom $T$. pseudonana and pennate $P$. tricornutum and give opportunity to analyse many processes on molecular level. Therefore, today three genes of diadinoxanthin deepoxidase have been identified in $P$. tricornutum. PtVDE gene is similar to deepoxidase in violaxanthin cycle in plants and has different length (Tab. 3) and sequences from PtVDL1 and PtVDL2 $[19,20]$. The aim of our study was application of the molecular methods for amplification of these three genes (PtVDE, PtVDL1 and PtVDL2) by PCR methods, to obtain the enzymes by expression of these genes in E. coli system and finally - isolation and analysis of the enzymes activity [20].

Table 3: Comparison of genes and protein size, and activity of PtVDE and PtVDL2 measured by xantophylls level (HPLC method) (modified Bojko et al. 2013 [20]).

\begin{tabular}{|c|c|c|c|}
\hline & PtVDE & PtVDL2 \\
\hline \multicolumn{2}{|c|}{ Gene size [bp] } & 1314 & 1668 \\
\hline \multicolumn{2}{|c|}{ Protein size [kDa] } & 49 & 60 \\
\hline \multirow{3}{*}{ 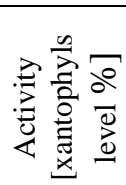 } & Violaxanthin & 94 & 87 \\
\hline & Anteraxanthin & 2 & 5 \\
\hline & Zeaxanthin & 4 & 8 \\
\hline
\end{tabular}

We obtained all three deepoxidases genes from P. tricornutum and finally PtVDE and PtVDL2 active enzymes. Differences in domain structure of PtVDE and PtVDL2 [19] suggest, that the enzymes could have different function and localization. The first PtVDE, relative to plant deepoxidase, can take part in conventional xantophyll cycle (deepoxidation of violaxanthin (Vx) by intermediate anteraxanthin (Ax) and final product - zeaxanthin (Zx)), but PtVDL2 can be more specialized in the chromist-specific diadinoxanthin cycle. Our results confirm different genes and enzymes size and show different activity of obtained deepoxidases in the presence of Vx as the substrate (Tab. 3). PtVDL2 converts Vx faster than PtVDE. The results obtained in model system suggest that PtVDE and PtVDL2 could be different in functions in diatoms.

\section{The fourth face - Treasury bioactive compounds in diatoms}

In recent years, researchers have become increasingly interested in bioactive compounds identified in diatoms. The main groups of them are omega-3 polyunsaturated fatty acids and carotenoids such as fucoxanthin, diadinoxanthin and diatoxanthin (Fig. 3 on the left). The bioactive compounds show many properties that can be beneficial to the human health.

Fx, the main pigment present in diatoms thylakoid in the light harvesting complexes, has antioxidant properties and can act under anoxic condition, what is unique among carotenoids [21]. Fx shows anti-cancer, anti-obesity and antidiabetic activities [22, 23, 24, 25, 26] and can inhibit inflammatory response [27]. Ddx and Dtx protect the photosynthetic apparatus against oxidative stress generated by high light [28]. These pigments are considered as compounds of sunscreens [29]. Furthermore, studies on Ddx have shown that it can inhibit the expression of pro-inflammatory cytokines in murine cells [30]. The analysis of beneficial properties of Ddx and Dtx for animals and human have not been studied extensively so far, because the pigments are presented in diatoms only in low level and their isolation and purification are complicated. 

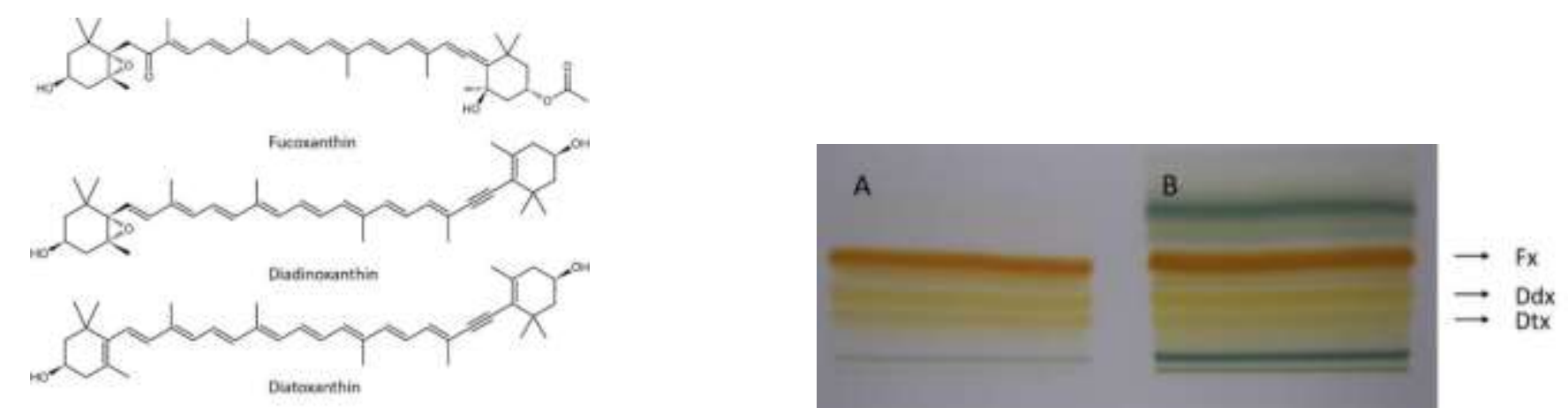

Fig. 3: Chemical formulas of carotenoid (on the left). HP-TLC plate after pigments separation. (A) when column pre-separation step was applied, (B) when no column pre-separation was applied (modified Tokarek et al. 2016 [33]) (on the right).

One of the central purpose subjects of our studies was isolation and purification these three xanthophylls and $P$. tricornutum is useful source of them [31, 32]. The Fx and Ddx were extracted from darkened diatoms culture, whereas Dtx from the culture treated with intensive illumination for 1.5 hour. After extraction we applied additional column preseparation step (HP-TLC, conducted by solvent containing ether) to separate of carotenoids from chlorophylls (Fig. 3 on the right). Our modification of the earlier isolation protocol of diatoms pigment increased the purity of Fx, Ddx and Dtx for biochemical studies (Tab. 4)[33].

Table 4: Purification procedure yield and purity of obtained pigments (modified Tokarek et al. 2016 [33]).

\begin{tabular}{|l|l|l|}
\hline Carotenoid & $\begin{array}{c}\text { Pigment purity } \\
{[\%]}\end{array}$ & $\begin{array}{c}\text { Purification yield } \\
{[\boldsymbol{\mu m o l}]}\end{array}$ \\
\hline Fx & $0.177( \pm 0.012)$ & $99.293( \pm 0.142)$ \\
\hline Ddx & $0.031( \pm 0.005)$ & $97.61( \pm 0.686$ \\
\hline Dtx & $0.0048( \pm 0.0006)$ & $89.002( \pm 1.004)$ \\
\hline
\end{tabular}

The high purity of isolated carotenoids allowed us to check the anti-cancer effect of these pigments. Fx in liposomes (made of phosphatidylcholine) were administered into the culture of colorectal adenocarcinoma cell line. Our finding provide the strong evidence that Fx-containing liposomes lowered the viability of tested cancer cell line and that this effect was the strongest at the highest Fx tested concentration [34].

Second group of bioactive compounds specific for diatoms are long-chain polyunsaturated fatty acids (LCPUFA). Polyunsaturated fatty acids (PUFA) are recommended in prevention from many diseases such as cardiovascular and inflammatory diseases, cancer, brain disorders, obesity, diabetes, autoimmune diseases and are important in fetus and infant development. PUFAs can be classified into two main groups: omega-6 (n-6) and omega3 (n-3) families, depending of the first double bound. Balanced diet should include both omega-3 and omega-6 fatty acids. Unfortunately, the ratio of omega-6/omega-3 FA of modern diet is very high and should be reduced. Omega-3 LC-PUFAs, particularly eicosapentaenoic acid (EPA) and docosahexaenoicacid (DHA) present in membranes of erythrocytes, neutrophils, monocytes and liver cells, therefore play essential role in human nutrition [35, 36]. Currently the main source of EPA and DHA are marine fishes, however diatoms, the primary producers, represent new and promising alternative source of LC-PUFAs. 


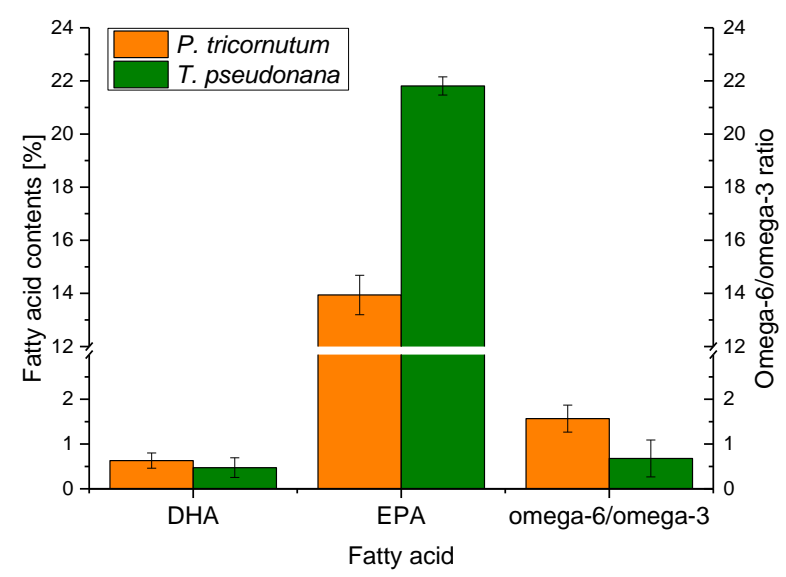

Fig. 4: Fatty acid composition of diatoms (modified Bojko et al. 2017 [8], 2017 [37]).

We grew the diatoms $P$. tricornutum and T. pseudonana under specified conditions, analysed the fatty acid compounds (by static headspace gas chromatography with flame ionization detector, HSGC-FID) and thus confirmed the presence of DHA, dominant amount of EPA and very low omega-3/omega-6 FA ratio (Fig. 4)[4, 8, 37, 38]. The results suggest, that the marine diatoms could be considered as a potential source for industrial production of omega-3 LCPUFAs.

\section{The fifth face - Diatoms a rich source of biofuels}

It is commonly known that diatoms show high potential to synthesis and accumulation of lipids, which can be used for production of biodiesel. The biodiesel production by diatoms has not been examined in our laboratory. Many marine and freshwater diatoms species were examined for their capability to produce biomass and lipids, especially triglyceride levels, as preferential source of biofuels [39 - 42]. Two centric marine diatoms Thalassiosira weissflogi and Cyclotella cryptica were selected as good candidates for oil production. Furthermore, the nitrogen limitation led to increase de novo synthesis of triacyloglycerols up to $88 \%$ of total glycerolipids [41]. The screening of common freshwater diatoms, selected Cyclotella managhiniana and Aulacoseria granulate as producers of the highest levels of lipids [39]. Generally, the rate of lipids accumulation under laboratory conditions is substantially improved under silicon limitation. [40]. Biofuels production by microalgae have been examined in continuous culture in outdoor photobioreactors. The outdoor biofuels production will have regional character and it will be needed to select diatoms optimally adapted to different outdoor conditions. For example P. tricornutum (diatom growing well under laboratory conditions [7]) was examined in quasi outdoor steady-stare continuous culture [43]. Stable year-round production of microalgae lipids for biofuel production requires application of two selected oleaginous diatom species - Fistulifera solaris for cultivation in out-door bioreactor from spring to autumn and Mayamaea sp. JPCCCTDA 0820 in winter [44] in Japan. The physiological and metabolic response of diatoms to different environment condition are the important factors in outdoor production systems.

\section{Conclusion}

Diatoms achieved ecological success by the ability to acclimation to changing environmental conditions (temperature, light, contamination by heavy metals) and show huge potential as bioremediating organisms. Their sequenced genome give possibility to analyse the molecular mechanisms of acclimation, together with investigations of diatom's physiology and biochemistry. Unique fatty acids composition (specially EPA) and carotenoids profile (Fx, Ddx and Dtx) different from vascular plants thereby suggest, that diatoms can be used for supplementation of diet production.. Moreover, the possibility of increasing the amount of triacylglycerols in cellular mass allow to consider the diatoms as potential source of biofuels. 


\section{Acknowledgements}

We would like to thank Anna Kowalczyk, Stanisław Listwan and Wiktor Tokarek for their technical help and contribution in preparing the paper.

\section{References}

[1] P. G. Falkowski, R. T. Barber, V. Smetacek, "Biochemical controls and feedbacks on ocean primary production," Science, vol. 281, pp. $200-206,1998$.

[2] E. V. Armbrust, "The life of diatoms in the world's ocean," Nature, vol. 459, no. 14, pp. 185 - 192, 2009.

[3] A. M. Pyszniak, S. P. Gibbs, "Immunocytochemical localization of photosystem I and the fucoxanthin-chlorophyll a/c light-harvesting complex in the diatom Pheodactylum tricornutum," Protoplasma, vol. 166, pp. 208 - 2017, 1992.

[4] M. Bojko, M. Olchawa-Pajor, M. Chyc, A. Kowalczyk, D. Latowski, "Diatoms as a source of polyunsaturated fatty acids essential to the human diet," in New Biotechnology vol. 33 (Supplement), pp. S160 Abstract of 17th European Congress on Biotechnology 3 - 6.07.2016, Krakow, Poland.

[5] W.I. Gruszecki, K. Strzałka, "Carotenoids as modulator of lipid membrane physical properties," Biochim. Biophys. Acta., vol. 1740, pp. 108 - 115, 2005.

[6] M. Bojko, M. Olchawa-Pajor, R. Goss, S. Schaller-Lauder, K. Strzałka, D. Latowski, "Diadinoxanthin deepoxidation as important factor in the short-term stabilization of diatom photosynthetic membranes exposed to different temperature," Plant Cell Environ., vol. 42, pp. 2019, 42:1270 - 1286, 2019.

[7] M. Bojko, K. Brzostowska, P. Kuczyńska, D. Latowski, M. Olchawa-Pajor, W. Krzeszowiec, A. Waloszek, K. Strzałka, "Temperature effect on growth, and selected parameters of Phaeodactylum tricornutum in batch cultures," Acta Biochim. Pol., vol. 60, no. 4, pp. 861 - 864, 2013.

[8] M. Bojko, M. Olchawa-Pajor, M. Chyc, R. Goss, S. Schaller-Laudel, D. Latowski, "Acclimatization of Thalassiosira pseudonana photosynthetic membranes to environmental temperature change," in Proceedings of the $3^{\text {rd }}$ World Congress on New Technologies (NewTech'17) Rome, Italy - June 6 - 8, 2017 Paper No. 120, 2017.

[9] E. Warkiewicz, A. Strączek, D. Latowski, M. Bojko, "Acclimatization of diatom Phaeodactylum tricornutum to long-term environmental temperature and light intensity changes," in Proceedings of the 4th World Congress on New Technologies (NewTech'18) Madrid, Spain - August 19 - 21, 2018 Paper No. ICEPR 146, 2018.

[10] A. Alvarez, J. M. Saez, J. S. Davila Costa, V. L. Colin, M. S. Fuentes, S. A. Cuozzo, C. S. Benimeli, M. A. Polti, M. J. Amoroso, "Actinobacteria: Current research and perspectives for bioremediation of pesticides and heavy metals," Chemosphere, vol. 166, pp. $41-62,2017$.

[11] J. Wang, C. Chen, "Bio sorbents for heavy metals removal and their future," Biotechnol Adv., vol. 27, pp. 195 226, 2009.

[12] R.B. Meagher, "Phytoremediation of toxic elemental and organic pollutants," Cur. Opin. Plant Biol. vol. 3 , pp. 153 $-62,2000$.

[13] N. Yoshida, R. Ikeda, T. Okuno, "Identification and characterization of heavy metal-resistant unicellular alga isolated from soil and its potential for phytoremediation," Bioresour Technol. vol. 97, pp.1843 - 9, 2006.

[14] W. Tokarek, S. Listwan, K. Krawczyk, K. Pajdzik, Z. Porębska, K. Stopa, M. Wasilewska, D. Latowski, "The influence of heavy metals on Phaeodactylum tricornutum growth - a preliminary study," in Contemporary Problems of Power Engineering and Environmental Protection 2016, Edited by Krzysztof Pikoń and Lucyna Czarnowska, Published by Department of Technologies and Installations for Waste Management, pp. 161 - 167, 2016.

[15] S. Listwan, W. Tokarek, K. Kleszcz, M. Chowaniec, Z. Porębska, K. Krawczyk, M. Bojko, D. Latowski, "Phaeodactylum tricornutum as a potential phytoremediator of sea and fresh waters," in Proceedings of the $4^{\text {rd }}$ World Congress on New Technologies (NewTech'18) Madrid, Spain - August 19 - 21, 2018 Paper No. ICEPR 147.

[16] G. F. Deng, T. W. Zhang, L. M. Yang, and Q. Q. Wang, "Studies of bio uptake and transformation of mercury by a typical unicellular diatom Phaeodactylum tricornutum," Chinese Sci. Bull., vol. 58, no. 2, pp. 256 - 265, 2013.

[17] P. Jahns, D. Latowski, K. Strzałka, "Mechanism and regulation of violaxanthin cycle: The role of antenna proteins and membrane lipids," Biochim Biophys Acta, vol. 1787, pp.3 - 14, 2009. 
[18] R. Goss, T. Jakob, "Regulation and function of xantophyll cycle-dependent photoprotection in algae," Photosynth Res, vol. 106, pp. $103-122,2010$.

[19] S. Coesel, M. Obornik, J. Varela, A. Falciatore, C. Bowler, "Evolutionary origins and functions of the carotenoid biosynthetic pathway in marine diatoms," PLos One, vol. 3, no. 8, pp. e2896, 2008.

[20] M. Bojko, M. Olchawa-Pajor, U. Tuleja, P. Kuczyńska, W. Strzałka, D. Latowski, K. Strzałka, "Expression of three diadinoxanthin de-epoxidase genes of Phaeodacylum tricornutum in Escherichia coli Origami b and BL21 strain," Acta Biochim. Pol., vol. 60, no. 4, pp. 857 - 86, 2013.

[21] T. Nomura, M. Kikuchi, A. Kubodera, Y. Kawakami, "Proton-donative antioxidant activity of fucoxanthin with 1,1-diphenyl-2-picrylhydrazyl (DPPH)," Biochem. Mol. Biol. Int., vol. 42, pp. 361 - 370, 1997.

[22] N. D’Orazio, E. Gemello, M. A. Gammone, M. de Girolamo, C. Ficoneri, G. Riccioni, "Fucoxanthin: a treasure from the sea," Mar. Drugs, vol. 10, pp. $604-616,2012$.

[23] K. Mikami, M. Hosokawa, "Biosynthetic pathway and health benefits of fucoxanthin, an algae-specific xanthophyll in brown seaweeds," Int. J. Mol. Sci., vol. 14, pp. 13763 - 13781, 2013.

[24] S. R. Kumar, M. Hosokawa, K. Miyashita, "Fucoxanthin: a marine carotenoid exerting anti-cancer effects by affecting multiple mechanisms," Mar. Drugs, vol. 11, pp. 5130 - 5514, 2013.

[25] S. Z. Moghadamtousi, H. Karimian, R. Khanabdali, M. Razavi, M. Firoozinia, K. Zandi, H. A. Kadir, "Anticancer and antitumor potential of fucoidan and fucoxanthin, two main metabolites isolated from brown algae," The Scientific World Journal, 2014, Article ID 768323.

[26] K. Miyashita, M. Hosokawa, "The beneficial health effects of fucoxanthin. In genomics, proteomics and metabolomics in nutraceuticals and functional foods," Eds. D. Bagchi, A. Swaroop, M. Bagchi, second edition, pp 122-134, 2015, John Wiley \& Sons, Ltd, Chichester, UK.

[27] J. H. Choi, N. H. Kim, S. J. Kim, H. J. Lee, S. Kim, "Fucoxanthin inhibits the inflammation response in paw edema model through suppressing MAPKs, Akt, and NFкB," J. Biochem. Mo. Toxicol., vol. 30, pp. 111 - 119, 2015.

[28] D. Latowski, P. Kuczyńska, K. Strzałka, "Xanthophyll cycle - a mechanism protecting plants against oxidative stress," Redox Report, vol. 16, pp. 78 - 90, 2011.

[29] G. Johnsen, P.A. Lysaa, K. Aamodt, "Sunscreen Compositions Comprising Carotenoids," Promar AS, assignee. US Patent US 2014, 8834855 B2.

[30] I. Konishi, M. Hosokawa, T. Sashima, T. Maoka, K. Miyashita, "Suppressive effects of alloxanthin and diatoxanthin from Halocynthia roretzi on LPS-induced expression of pro-inflammatory genes in RAW264.7 cells," J. Oleo Sci, vol. 57, pp. 181 - 189, 2008.

[31] Z. Cohen, "Chemicals from Microalgae," Z. Cohen ed, pp 149 - 160, 1990, Taylor \& Francis Ltd.

[32] B. E. Pfeil, B. Schoefs, C. Spetea, "Function and evolution of channels and transporters in photosynthetic membranes," Cell Mol. Life Sci., vol. 71, pp. 979 - 998, 2014.

[33] W. Tokarek, S. Listwan, J. Pagacz, P. Leśniak, D. Latowski, "Column chromatography as a useful step in purification of diatom pigments," Acta Biochim. Pol., vol. 64, no. 3, pp. 443 - 447, 2016.

[34] W. Tokarek, M. Kocemba, S. Listwan, A. Pisarek, P. Leśniak, J. Pagacz, K. Stalińska, D. Latowski, "Fucoxanthin algal biomolecule exerting beneficial health effects," in Book of Abstracts and Program of XII International Conference Biomolecules: Identification and Functions, 21-22.10.2016 Kraków, Poland, pp. 23.

[35] A. P. Simopoulos, "Evolutionary aspects of diet, the omega-6/omega-3 ratio and genetic variation:nutritional implications for chronic diseases," Biomedicine \& Pharmacotherapy, vol. 60, pp. 502 - 507, 2006.

[36] S. Bellou, I. E. Triantaphyllidou, D. Aggeli, A. M .Elazzazy, M. N. Baeshen, G. Aggelis, "Microbial oils as food additives: recent approaches for improving microbial oil production and its polyunsaturated fatty acid content," Current Opinion in Biotechnology, vol. 37, pp. 23 - 35, 2016.

[37] M. Bojko, M. Chyc, S. Listwan, D. Latowski, "Effect of environmental temperature on Pheodactylum tricornutum fatty acid composition," in Abstract Book of XLIV Winter School, Faculty of Biochemistry, Biophysics and Biotechnology, Jagiellonian University „, No stress no life”, 14-18.02.2017, Zakopane, Poland.

[38] M. Bojko, M. Olchawa-Pajor, M. Chyc, D. Latowski, "Alternative sources of different omega-3 polyunsaturated fatty acids," in Book of abstract of XLVII Winter School, Faculty of Biochemistry, Biophysics and Biotechnology, Jagiellonian University ,, The light site of the fors”, 11-15.02.2019, Zakopane, Poland. 
[38] J. M. Graham, L. E. Graham, S. B. Zulkyfly, B. F. Pfleger, S. W. Hoover, J. Yoshitani, "Freshwater diatoms as a source of lipids for biofuels," J. Ind. Microbiol. Biotechnol., vol. 39, pp. 419 - 428, 2012.

[40] M. Hildebrand, A. K. Davis, S. R. Smith, J. C. Traller, R. Abbriano, "The place of diatoms in the biofuels industry," Biofuels, vol. 3 no. 2, pp. 221 - 240, 2012.

[41] G. d'Ippolito, A. Sardo, D. Paris, F. M. Vella, M. G. Adelfi, P. Botte, C.Gallo, A. Fontana, "Potential of lipid metabolism in marine diatoms for biofuel production," Biotechnology for biofuels, pp. 1-10, 2015.

[42] J. K. Wang, M. Seibert, "Prospects for commercial production od diatom, "Biotechnology for Biofuels," vol. 10, pp. $1-16,2017$.

[43] A. S. Miron, M. C. C. Garcia, A. C. Gomez, F. G. Camacho, E. M. Grima, Y. Chisti, "Shear stress tolerance and biochemical characterization of Phaeodactylum tricornutum in quasi steady-state continuous culture in outdoor photobioreactors," Biochem. Engin J., vol. 16, pp. 287 - 297, 2003.

[44] M. Matsumoto, D. Nojima, T. Nonoyama, K. Ikeda, Y. Maeda, T. Yoshino, T. Tanaka, "Outdoor cultivation of marine diatoms for year-round production of biofuels," Marine Drugs, vol. 15, no. 94, 2017. 